\title{
New Access to Pyrano[2,3-c]pyrazole-3-carboxylates via Domino Four-Component Reaction and Their Antimicrobial Activity
}

\author{
Muhammad Siddiq Maarop ${ }^{1}$, Fatin Nur Ain Abdul Rashid ${ }^{1}$, Mohd Fazli Mohammat ${ }^{2,}$, Zurina \\ Shaameri ${ }^{2}$, Saiful Azmi Johari ${ }^{3}$, Mazurah Mohamed Isa ${ }^{3}$, and Anis Low Muhammad Low ${ }^{4}$ \\ ${ }^{1}$ Department of Chemistry, Faculty of Applied Sciences, Universiti Teknologi MARA, \\ UiTM Shah Alam, 40450 Shah Alam, Selangor, Malaysia \\ ${ }^{2}$ Organic Chemistry Laboratory, Institute of Science, Universiti Teknologi MARA, \\ Kampus Puncak Alam, 42300 Bandar Puncak Alam, Selangor, Malaysia \\ ${ }^{3}$ Antimicrobial Laboratory, Anti-Infective Branch, Bioactivity Programme, Natural Products Division, \\ Forest Research Institute Malaysia (FRIM), 52109 Kepong, Selangor, Malaysia \\ ${ }^{4}$ Atta-ur-Rahman Institute (AURINS), Universiti Teknologi MARA, Kampus Puncak Alam, 42300 Bandar Puncak Alam, \\ Selangor, Malaysia
}

\section{* Corresponding author:}

email:mohdfazli@salam.uitm.edu.my

Received: October 10, 2018

Accepted: April 4, 2019

DOI: $10.22146 / \mathrm{ijc} .39566$

\begin{abstract}
A library of some novel classes of pyrano[2,3-c]pyrazole-3-carboxylates was synthesized by employing uncatalyzed domino four-component reaction using diethyloxaloacetate, hydrazine hydrate, aldehydes and malononitrile in refluxing of ethanol-acetic acid solvent systems. Series of domino reactions involving of pyrazolone formation, Michael addition, and Thorpe-Ziegler cyclization reaction managed to produce the cyclized products from moderate to excellent yield. This protocol provides a reliable, general and salient procedure for the title compound using a one-pot approach. Preliminary biological screening unveiled limited potentials of this class of compounds for antimicrobial lead compound due to its limited solubility properties.
\end{abstract}

Keywords: four-component reactions; pyrano[2,3-c]pyrazole-3-carboxylate; diethyloxaloacetate

\section{- INTRODUCTION}

Isomeric pyranopyrazole structure includes pyrano[2,3-c]pyrazole, pyrano[4,3-c]pyrazole, pyrano[3, 2-c]pyrazole and pyrano[3,4-c]pyrazole (Fig. 1). Functionalized pyrano[2,3-c]pyrazoles are the most explored and widely studied and displayed significant roles in pharmaceutical fields. It possesses many interesting biological activities varying from antimicrobial [1], analgesic [2], vasodilator [3], anticancer [4], anti-inflammatory [5], inhibitors of human Chkl kinase [6], antifungicidal [7] and also as biodegradable agrochemicals [8].

The construction of pyrano[2,3-c]pyrazole structures has been established through different modes of multicomponent reactions (MCRs) either in two-, three- or four-component reactions [9-11]. MCRs are considered convergent one-pot reaction protocol involving two or more of simple yet different starting materials to provide highly complex materials or archetypical molecules with high variability. In addition,<smiles>C1=COc2[nH]ncc2C1</smiles>

Pyrano[2,3-c]pyrazole<smiles>c1cc2nncc-2co1</smiles>

Pyrano[4,3-c]pyrazole<smiles>c1coc2cnnc-2c1</smiles><smiles>c1cc2cnnc-2co1</smiles>

Fig 1. Structures of isomeric pyranopyrazole 
MCR approaches also offered multiple advantages including the elimination of complicated purification operations, the use of readily available flexible building blocks as well as solvent and reagent economical purposes [12].

Currently, most MCRs toward constructing the pyranopyrazole ring skeleton involved the reagents of hydrazines, $\beta$-ketoesters, aldehydes, and the active methylene nitriles. Interestingly, with regards to our literature searches, there is only a single report that successfully employed diethyl oxaloacetate as the source of the active methylene group. This one-pot reaction was successfully performed by Gein et al. during the synthesis of ethyl 6-amino-4-aryl-5cyano-1,4-dihydropyrano[2,3c] pyrazole-3-carboxylates, using a four-component twoparallel reaction manner [13]. Previously, diethyl oxalacetate was reported as a non-common source of the active methylene group in most of the MCRs for bearing two active ester groups that prone to undergo different multiple substitution reactions [14].

Initially, replicating four-component two-parallel reaction manner as reported by Gein et al. [13], we managed to synthesize a library of some novel dihydropyrano[2,3-c]pyrazoles-3-carboxylates, but in reasonable yield. Nevertheless, upon changing the original reaction protocol to domino type reaction manner furnished the title compounds and their derivatives from moderate to excellent yields. This library of compounds was then subjected to antimicrobial study as part of our endeavor on the screening of biologically active heterocyclic type compounds [15-18].

\section{- EXPERIMENTAL SECTION}

\section{Materials}

All reagents and starting materials were purchased from Sigma-Aldrich Co. and Merck Chemical Co. Thin layer chromatography (TLC) was performed using aluminum precoated sheets (Merck Kieselgel $60 \mathrm{GF}_{254}$, $0.25 \mathrm{~mm}$ thick) and was visualized with an ultraviolet lamp (254 and $365 \mathrm{~nm}$ ). Bacterial species for in vitro antibacterial test were Escherichia coli (E.C), Salmonella typhimurium (S.T), and Proteus vulgaris (P.V) as Gramnegative (-ve) and Staphylococcus aureus (S.A),
Staphylococcus cohnii (S.C) and Staphylococcus haemolyticus (S.H) as Gram-positive.

\section{Instrumentation}

Melting points were determined on an automatic FP62 melting point apparatus from Mettler Toledo and are uncorrected. ${ }^{1} \mathrm{H}$ and ${ }^{13} \mathrm{C}$-NMR spectra were recorded on JOEL NMR Spectrometer instrument operating at $400 \mathrm{MHz}$ at room temperature, in $\mathrm{CDCl}_{3}$ or DMSO solutions. Chemical shift values are given in $\delta$ units $(\mathrm{ppm})$ relative to TMS as an internal standard. IR spectra (4000-400 $\mathrm{cm}^{-1}$ ) were recorded on Varian Excalibur 3100 FT-IR spectrometer, using ATR. CHNS was performed on Flash Elemental Analyzer 110 series.

The antimicrobial test were performed using Well Diffusion Method, Sample concentration: $5.0 \mathrm{mg} / \mathrm{mL}$ (50\% DMSO prepared in $2 \mathrm{~mL}$ ), Incubation temperature: $37^{\circ} \mathrm{C}$, Positive Control: Streptomycin, Chloramphenicol $(500 \mu \mathrm{g} / \mathrm{mL})$, Negative Control: 50\% DMSO.

\section{Procedure}

\section{General procedure for the synthesis of pyrano- pyrazole 5a-5t. (Method A)}

To a solution of diethyloxalacetate sodium salt (5.5 mmol) in $20 \mathrm{~mL}$ ethanol, 35\% hydrazine solution $(5.5 \mathrm{mmol})$ and $1 \mathrm{~mL}$ of acetic acid were added and refluxed for $15 \mathrm{~min}$. Then, carbonyl compound ( $5 \mathrm{mmol}$ ) and malononitrile $(5 \mathrm{mmol})$ were added to the reaction mixture, and the reflux continued for an additional $15 \mathrm{~min}$. The reaction mixture was left to cool, and the resulting solid was filtered off, washed with water.

(5a) Ethyl 6-amino-5-cyano-4-phenyl-1,4-dihydro pyrano[2,3-c]pyrazole-3-carboxylate. Following the above mentioned procedure, $5 \mathrm{a}$ was isolated as a white solid (82\%). m.p 226-227 ${ }^{\circ} \mathrm{C}$. IR spectrum, $v, \mathrm{~cm}^{-1}: 3388$ $\left(\mathrm{NH}_{2}\right), 3218(\mathrm{NH}), 2199(\mathrm{CN}), 1716$ (COOEt), 1651 $(\mathrm{C}=\mathrm{C}) .{ }^{1} \mathrm{H}-\mathrm{NMR}$ (400 MHz, DMSO): 7.26-7.22 (m, 2H), 7.17-7.13 (m, 1H), $7.06(\mathrm{~m}, 2 \mathrm{H}), 6.99(\mathrm{~s}, 2 \mathrm{H}), 4.71(\mathrm{~s}$, $1 \mathrm{H}), 4.06-4.01(\mathrm{~m}, 2 \mathrm{H}), 1.01-0.98(\mathrm{t}, 3 \mathrm{H}) .{ }^{13} \mathrm{C}-\mathrm{NMR}$ (100 MHz, DMSO): ${ }^{13} \mathrm{C}-\mathrm{NMR}$ (100 MHz, DMSO,): $160.5\left(\mathrm{CNH}_{2}\right), 158.6(\mathrm{C}=\mathrm{O}), 156.1(\mathrm{CNH}), 145.4$ (quat. Ar C), 129.5 (C=N), 128.7 (Ar C), 127.8 (ArC), 127.1 (Ar C), $120.8(\mathrm{CN}), 104.1$ (quat. C), $61.3\left(\mathrm{CH}_{2}\right), 58.3$ (quat. 
C), $37.5(\mathrm{CH}), 14.3\left(\mathrm{CH}_{3}\right)$.Anal.calc. (\%) for $\mathrm{C}_{16} \mathrm{H}_{14} \mathrm{~N}_{4} \mathrm{O}_{3}$, C 61.93, H 4.55 Found: C 62.04; H 4.52.

(5b) Ethyl 6-amino-5-cyano-4-(4-methoxyphenyl)-1,4dihydropyrano[2,3-c]pyrazole-3-carboxylate. Following the above mentioned procedure, $5 \mathrm{~b}$ was isolated as a white solid (65\%), m.p 235-236 -C. IR spectrum, $v, \mathrm{~cm}^{-1}: 3429$ $\left(\mathrm{NH}_{2}\right), 33180(\mathrm{NH}), 2195(\mathrm{CN}), 1717$ (COOEt), 1633 $(\mathrm{C}=\mathrm{C}) .{ }^{1} \mathrm{H}-\mathrm{NMR}(400 \mathrm{MHz}, \mathrm{DMSO}):$ 7.02-6.97 (m, 2H), $6.95(\mathrm{~s}, 2 \mathrm{H}), 6.81-6.77(\mathrm{~m}, 2 \mathrm{H}), 4.65(\mathrm{~s}, 1 \mathrm{H}), 4.08-4.03(\mathrm{~m}$, 2H), $3.66(\mathrm{~s}, 3 \mathrm{H}), 1.06-1.03(\mathrm{t}, 3 \mathrm{H}) .{ }^{13} \mathrm{C}-\mathrm{NMR}(100 \mathrm{MHz}$, DMSO): $160.4\left(\mathrm{CNH}_{2}\right), 158.7(\mathrm{C}=\mathrm{O}), 158.4(\mathrm{CNH}), 137.6$ (Ar C), 130.5 (Ar C), 128.8 (Ar C), 127.1 (Ar C), 120.9 (CN), 114.9 (quat. C), 114.1 (quat. C), 104.5 (quat. C), $61.3\left(\mathrm{CH}_{2}\right), 58.6$ (quat. C), $55.5\left(\mathrm{OCH}_{3}\right), 36.7(\mathrm{CH}), 14.3$ $\left(\mathrm{CH}_{3}\right)$.Anal.calc. (\%) for $\mathrm{C}_{17} \mathrm{H}_{16} \mathrm{~N}_{4} \mathrm{O}_{4}, \mathrm{C} 59.99, \mathrm{H} 4.74$ Found: C 54.59; H 3.51.

(5c) Ethyl 6-amino-5-cyano-4-(4-ethoxyphenyl)-1,4dihydropyrano[2,3-c]pyrazole-3-carboxylate. Following the above mentioned procedure, $5 \mathrm{c}$ was isolated as a yellowish solid (60\%), m.p $210-211^{\circ} \mathrm{C}$. IR spectrum, $v$, $\mathrm{cm}^{-1}$ : $3413\left(\mathrm{NH}_{2}\right), 3290(\mathrm{NH}), 2206(\mathrm{CN}), 1740$ (COOEt), 1660 (C=C). ${ }^{1} \mathrm{H}-\mathrm{NMR}$ (400 MHz, DMSO): 6.95-6.92 (m, $4 \mathrm{H}), 6.78-6.76(\mathrm{~m}, 2 \mathrm{H}), 4.64(\mathrm{~s}, 1 \mathrm{H}), 4.05(\mathrm{~m}, 2 \mathrm{H}), 3.91$ $(\mathrm{m}, 2 \mathrm{H}), 1.25(\mathrm{t}, 3 \mathrm{H}), 1.04(\mathrm{t}, 3 \mathrm{H}) .{ }^{13} \mathrm{C}-\mathrm{NMR}(100 \mathrm{MHz}$, DMSO): $160.4\left(\mathrm{CNH}_{2}\right), 158.7(\mathrm{C}=\mathrm{O}), 157.6$ (quat. $\left.\mathrm{C}\right)$, 137.4 (quat. Ar C), 129.4 (quat. C), 128.8 (Ar C), 120.9 (CN), 114.5 (Ar C), 104.5 (quat. C), $63.4\left(\mathrm{CH}_{2}\right), 61.3$ $\left(\mathrm{CH}_{2}\right), 58.6$ (quat. C), $36.71(\mathrm{CH}), 15.19\left(\mathrm{CH}_{3}\right), 14.3$ $\left(\mathrm{CH}_{3}\right)$.Anal.calc. (\%) for $\mathrm{C}_{18} \mathrm{H}_{18} \mathrm{~N}_{4} \mathrm{O}_{4}, \mathrm{C} 61.01, \mathrm{H} 5.12$ Found: C 59.53; H 4.93.

(5d) Ethyl 6-amino-5-cyano-4-(4-ethylphenyl)-1,4dihydropyrano[2,3-c]pyrazole-3-carboxylate. Following the above mentioned procedure, $5 \mathrm{~d}$ was isolated as a yellowish solid $69 \%$, m.p $212-213{ }^{\circ} \mathrm{C}$. IR spectrum, $v, \mathrm{~cm}^{-}$ ': $3433\left(\mathrm{NH}_{2}\right), 3155(\mathrm{NH}), 2194(\mathrm{CN}), 1727$ (COOEt), $1631(\mathrm{C}=\mathrm{C}) .{ }^{1} \mathrm{H}-\mathrm{NMR}$ (400 MHz, DMSO): 7.08 (dd, 2H), 6.97-6.94 (m, 4H), $4.66(\mathrm{~s}, 1 \mathrm{H}), 4.06-4.01(\mathrm{~m}, 2 \mathrm{H}), 2.51$ (m, 2H), $1.65(\mathrm{t}, 3 \mathrm{H}), 1.02(\mathrm{t}, 3 \mathrm{H}) .{ }^{13} \mathrm{C}-\mathrm{NMR}(100 \mathrm{MHz}$, DMSO): $160.5\left(\mathrm{CNH}_{2}\right), 158.7(\mathrm{C}=\mathrm{O}), 156.1(\mathrm{CNH}), 142.8$ (quat. Ar C), 142.5 (quat. Ar C), 129.4 (quat. C), 128.1 ( $\mathrm{Ar}$ C), 127.7 (Ar C), 120.9 (CN), 104.3 (quat. C), $61.3\left(\mathrm{CH}_{2}\right)$, 58.4 (quat. C), $37.1(\mathrm{CH}), 28.1\left(\mathrm{CH}_{2}\right), 16.0\left(\mathrm{CH}_{3}\right), 14.2$
$\left(\mathrm{CH}_{3}\right)$.Anal.calc. (\%) for $\mathrm{C}_{18} \mathrm{H}_{18} \mathrm{~N}_{4} \mathrm{O}_{3}, \mathrm{C} 63.89$, H 5.36 Found: C 64.46, H 5.41.

(5e) Ethyl 6-amino-5-cyano-4-(4-nitrophenyl)-1,4-dihy dropyrano[2,3-c]pyrazole-3-carboxylate. Following the above mentioned procedure, $5 \mathrm{e}$ was isolated as a yellowish solid 75\%, m.p 235-237 -C. IR spectrum, $v, \mathrm{~cm}^{-1}: 3357$ $\left(\mathrm{NH}_{2}\right), 3155(\mathrm{NH}), 2195(\mathrm{CN}), 1723$ (COOEt), 1631 $(\mathrm{C}=\mathrm{C}) .{ }^{1} \mathrm{H}-\mathrm{NMR}(400 \mathrm{MHz}, \mathrm{DMSO}): 8.14-8.11(\mathrm{~m}, 2 \mathrm{H})$, 7.37-7.33 (m, 2H), 7.15 (s, 2H), 4.92 (s, 1H), 4.05-4.00 $(\mathrm{m}, 2 \mathrm{H}), 1.01-0.97 \quad(\mathrm{t}, 3 \mathrm{H}) .{ }^{13} \mathrm{C}-\mathrm{NMR}(100 \mathrm{MHz}$, DMSO): $160.7\left(\mathrm{CNH}_{2}\right), 158.4(\mathrm{C}=\mathrm{O}), 152.7(\mathrm{CNH})$, 146.7 (quat. Ar C), 129.5 (quat. C), 129.3 (Ar C), 124.1 (Ar C), $120.4(\mathrm{CN}), 102.7$ (quat. C), $61.5\left(\mathrm{CH}_{2}\right), 57.0$ (quat. C), $37.1(\mathrm{CH}), 14.3\left(\mathrm{CH}_{3}\right)$.Anal.calc. (\%) for $\mathrm{C}_{16} \mathrm{H}_{13} \mathrm{~N}_{5} \mathrm{O}_{5}, \mathrm{C} 54.09, \mathrm{H} 3.69$ Found: C 54.22, H 3.60.

(5f) Ethyl 6-amino-5-cyano-4-(3-nitrophenyl)-1,4-dihy dropyrano[2,3-c]pyrazole-3-carboxylate. Following the above mentioned procedure, $5 \mathrm{f}$ was isolated as a yellowish solid 83\%, m.p $224-225^{\circ} \mathrm{C}$. IR spectrum, $v, \mathrm{~cm}^{-1}: 3432$ $\left(\mathrm{NH}_{2}\right), 3183(\mathrm{NH}), 2189(\mathrm{CN}), 1713$ (COOEt), 1635 $(\mathrm{C}=\mathrm{C}) .{ }^{1} \mathrm{H}-\mathrm{NMR}(400 \mathrm{MHz}, \mathrm{DMSO}): 8.07-8.05(\mathrm{~m}, 1 \mathrm{H})$, $7.93(\mathrm{~m}, 1 \mathrm{H}), 7.57(\mathrm{~m}, 2 \mathrm{H}), 7.16(\mathrm{~s}, 2 \mathrm{H}), 4.97(\mathrm{~s}, 1 \mathrm{H}), 4.03$ $(\mathrm{m}, 2 \mathrm{H}), 1.00(\mathrm{t}, 3 \mathrm{H}) .{ }^{13} \mathrm{C}-\mathrm{NMR}(100 \mathrm{MHz}, \mathrm{DMSO})$ : $160.7\left(\mathrm{CNH}_{2}\right), 158.4(\mathrm{C}=\mathrm{O}), 155.9(\mathrm{CNH}), 148.1$ (quat. Ar C), 147.5 (quat. Ar C), 134.9 (Ar C), 130.5 (Ar C), 129.8 (quat. C), 122.4 ( $\mathrm{Ar} \mathrm{C}), 120.5(\mathrm{CN}), 102.8$ (quat. C), $61.5\left(\mathrm{CH}_{2}\right), 57.3$ (quat. C), $36.9(\mathrm{CH}), 14.2$ $\left(\mathrm{CH}_{3}\right)$.Anal.calc. (\%) for $\mathrm{C}_{16} \mathrm{H}_{13} \mathrm{~N}_{5} \mathrm{O}_{5}, \mathrm{C} 53.92, \mathrm{H} 3.62$ Found: C 54.09; H 3.69.

(5g) Ethyl 6-amino-4-(4-bromophenyl)-5-cyano-1,4-dihy dropyrano[2,3-c]pyrazole-3-carboxylate. Following the above mentioned procedure, $5 \mathrm{~g}$ was isolated as a white solid 73\%, m.p $221-222{ }^{\circ} \mathrm{C}$. IR spectrum, $v, \mathrm{~cm}^{-1}: 3400$ $\left(\mathrm{NH}_{2}\right), 3174(\mathrm{NH}), 2189(\mathrm{CN}), 1770(\mathrm{COOEt}), 1637$ $(\mathrm{C}=\mathrm{C}) .{ }^{1} \mathrm{H}$ NMR (400 MHz, DMSO): 7.45-7.43 (m, 2H), $7.05(\mathrm{~s}, 2 \mathrm{H}), 7.04-7.01(\mathrm{~m}, 2 \mathrm{H}), 4.73(\mathrm{~s}, 1 \mathrm{H}), 4.07-4.02$ $(\mathrm{m}, 2 \mathrm{H}), 1.05-1.00 \quad(\mathrm{t}, 3 \mathrm{H}) .{ }^{13} \mathrm{C}-\mathrm{NMR}(100 \mathrm{MHz}$, DMSO): $160.5\left(\mathrm{CNH}_{2}\right), 158.6(\mathrm{C}=\mathrm{O}), 156.0(\mathrm{CNH})$, 144.8 (quat. Ar), 131.6 ( $\mathrm{ArC}$ ), 130.1 (Ar C), 129.6 (quat. C), $120.6(\mathrm{CN}), 103.5$ (quat. C), $61.4\left(\mathrm{CH}_{2}\right), 57.8$ (quat. C), $36.9(\mathrm{CH}), \quad 14.3 \quad\left(\mathrm{CH}_{3}\right)$.Anal.calc. $\quad(\%)$ for $\mathrm{C}_{16} \mathrm{H}_{13} \mathrm{BrN}_{4} \mathrm{O}_{3}, \mathrm{C} 49.38$, H 3.37 Found: C 48.40, H 3.18. 
(5h) Ethyl 6-amino-5-cyano-4-(4-hydroxyphenyl)-1,4dihydropyrano[2,3-c]pyrazole-3-carboxylate. Following the above mentioned procedure, $5 \mathrm{~h}$ was isolated as a white solid 57\%, m.p 217-218 ${ }^{\circ} \mathrm{C}$. IR spectrum, $v, \mathrm{~cm}^{-1}: 3406$ $\left(\mathrm{NH}_{2}\right), 3222(\mathrm{NH}), 2273(\mathrm{CN}), 1731$ (COOEt), 1650 $(\mathrm{C}=\mathrm{C}) .{ }^{1} \mathrm{H}-\mathrm{NMR}(400 \mathrm{MHz}, \mathrm{DMSO}): 9.23(\mathrm{~s}, 1 \mathrm{H}), 6.91(\mathrm{~s}$, $2 \mathrm{H}), 6.85-6.82(\mathrm{~s}, 2 \mathrm{H}), 6.63-6.60(\mathrm{~m}, 2 \mathrm{H}), 4.59(\mathrm{~s}, 1 \mathrm{H})$, 4.09-4.04 (m, 2H), 1.07-1.03 (t, 3H). ${ }^{13} \mathrm{C}-\mathrm{NMR}(100 \mathrm{MHz}$, DMSO): $160.3\left(\mathrm{CNH}_{2}\right), 158.7(\mathrm{C}=\mathrm{O}), 156.4(\mathrm{CNH}), 156.0$ (quat. $\mathrm{Ar} \mathrm{C}$ ), 135.9 (quat. $\mathrm{Ar} \mathrm{C}$ ), 129.4 (quat. C), 128.8 (Ar C), $120.9(\mathrm{CN}), 115.4(\mathrm{Ar} \mathrm{C}), 104.8$ (quat. C), $61.3\left(\mathrm{CH}_{2}\right)$, 58.8 (quat. C), $36.7(\mathrm{CH}), 14.3\left(\mathrm{CH}_{3}\right)$.Anal.calc. (\%) for $\mathrm{C}_{16} \mathrm{H}_{14} \mathrm{~N}_{4} \mathrm{O}_{4}, \mathrm{C} 58.89, \mathrm{H} 4.32$ Found: C 58.91, H 4.31.

(5i) Ethyl 6-amino-4-(3-bromo-4-hydroxyphenyl)-5cyano-1,4-dihydropyrano[2,3-c]pyrazole-3-carboxylate. Following the above mentioned procedure, $5 \mathrm{i}$ was isolated as a white solid $73 \%$, m.p $224-225^{\circ} \mathrm{C}$. IR spectrum, $v, \mathrm{~cm}^{-1}$ : $3402\left(\mathrm{NH}_{2}\right), 3320(\mathrm{NH}), 2196(\mathrm{CN}), 1712$ (COOEt), 1644 (C=C). ${ }^{1} \mathrm{H}-\mathrm{NMR}$ (400 MHz, DMSO): 10.12 (s, 1H), 7.15 $(\mathrm{m}, 1 \mathrm{H}), 7.00(\mathrm{~s}, 2 \mathrm{H}), 6.85-6.80(\mathrm{~m}, 2 \mathrm{H}), 4.63(\mathrm{~s}, 1 \mathrm{H}), 4.07$ (m, 2H), 1.09 (t, 3H). ${ }^{13} \mathrm{C}-\mathrm{NMR}$ (100 MHz, DMSO): 160.4 $\left(\mathrm{CNH}_{2}\right), 158.6(\mathrm{C}=\mathrm{O}), 155.8(\mathrm{CNH}), 153.1$ (quat. Ar C), 137.7 (quat. Ar C), 132.1 (Ar C), 129.5 (quat. C), 128.1 ( $\mathrm{Ar}$ C), 120.8 (CN), 116.7 (quat. Ar C), 109.1 (quat. C), 104.0 (quat. C), $61.4\left(\mathrm{CH}_{2}\right), 58.2$ (quat. C), $36.3(\mathrm{CH}), 14.3$ $\left(\mathrm{CH}_{3}\right)$.Anal.calc. (\%) for $\mathrm{C}_{16} \mathrm{H}_{13} \mathrm{BrN}_{4} \mathrm{O}_{4}, \mathrm{C} 47.43, \mathrm{H} 3.23$ Found: C 47.18, H 3.15.

(5j) Ethyl 6-amino-4-(3-chloro-4-hydroxyphenyl)-5cyano-1,4-dihydropyrano[2,3-c]pyrazole-3-carboxylate. Following the above mentioned procedure, $5 \mathrm{j}$ was isolated as a white solid 50\%, m.p 228-229 $\mathrm{C}$. IR spectrum, $v, \mathrm{~cm}^{-1}$ : $3406\left(\mathrm{NH}_{2}\right), 3319(\mathrm{NH}), 2202(\mathrm{CN}), 1701$ (COOEt), 1649 $(\mathrm{C}=\mathrm{C}) .{ }^{1} \mathrm{H}-\mathrm{NMR}(400 \mathrm{MHz}, \mathrm{DMSO}): 10.04(\mathrm{~s}, 1 \mathrm{H}), 7.00$ (s, 2H), 6.80-6.70 (m, 3H), $4.63(\mathrm{~s}, 1 \mathrm{H}), 4.4 .09-4.07$ (m, 2H), 1.08 (t, 3H). ${ }^{13} \mathrm{C}-\mathrm{NMR}$ (100 MHz, DMSO): 160.4 $\left(\mathrm{CNH}_{2}\right), 158.6(\mathrm{C}=\mathrm{O}), 152.1(\mathrm{CNH}), 152.1$ (quat. Ar C), 137.4 (quat. Ar C), 129.5 (quat. C), 129.2 (Ar C), 127.4 (Ar C), 120.8 (CN), 119.4 (quat. Ar C), 117.0 (Ar C), 104.0 (quat. C), $61.4\left(\mathrm{CH}_{2}\right), 58.2$ (quat. C), $36.4(\mathrm{CH}), 14.3$ $\left(\mathrm{CH}_{3}\right)$.Anal.calc. (\%) for $\mathrm{C}_{16} \mathrm{H}_{13} \mathrm{ClN}_{4} \mathrm{O}_{4}, \mathrm{C} 53.27, \mathrm{H} 3.63$ Found: C 53.02, H 3.57.

(5k) Ethyl 6-amino-5-cyano-4-(4-cyanophenyl)-1,4-dihy dropyrano[2,3-c]pyrazole-3-carboxylate. Following the above mentioned procedure, $5 \mathrm{k}$ was isolated as a yellowish solid 74\%, m.p 218-219 ${ }^{\circ} \mathrm{C}$. IR spectrum, $v, \mathrm{~cm}^{-1}: 3386$ $\left(\mathrm{NH}_{2}\right), 3214(\mathrm{NH}), 2233(\mathrm{CN}), 1713(\mathrm{COOEt}), 1650$ (C=C). ${ }^{1} \mathrm{H}-\mathrm{NMR}(400 \mathrm{MHz}, \mathrm{DMSO}):$ 7.74-7.72 (m, 2H), 7.28-7.12 (m, 2H), $7.12(\mathrm{~s}, 2 \mathrm{H}), 4.85(\mathrm{~s}, 1 \mathrm{H}), 4.05-4.00$ (m, 2H), 0.99 (t, 3H). ${ }^{13} \mathrm{C}-\mathrm{NMR}(100 \mathrm{MHz}, \mathrm{DMSO})$ : $160.6\left(\mathrm{CNH}_{2}\right), 158.4(\mathrm{C}=\mathrm{O}), 156.0(\mathrm{CNH}), 150.7$ (quat. Ar C), 132.9 (Ar C), 129.7 (quat. C), 129.0 (Ar C), 120.5 (CN), 119.3 (quat. Ar C), 102.8 (quat. C), $61.4\left(\mathrm{CH}_{2}\right)$, 57.2 (quat. C), $37.4(\mathrm{CH}), 14.3\left(\mathrm{CH}_{3}\right)$.Anal.calc. (\%) for $\mathrm{C}_{17} \mathrm{H}_{13} \mathrm{~N}_{5} \mathrm{O}_{3}, \mathrm{C} 60.89$, H 3.91 Found: C 62.02, H 3.83.

(5I) Ethyl 6-amino-5-cyano-4-(furan-2-yl)-1,4-dihydro pyrano[2,3-c]pyrazole-3-carboxylate. Following the above mentioned procedure, 51 was isolated as a yellowish solid 63\%, m.p 216-218 ${ }^{\circ} \mathrm{C}$. IR spectrum, $v, \mathrm{~cm}^{-1}: 3404$ $\left(\mathrm{NH}_{2}\right), 3298(\mathrm{NH}), 2192(\mathrm{CN}), 1713(\mathrm{COOEt}), 1644$ $(\mathrm{C}=\mathrm{C}) .{ }^{1} \mathrm{H}-\mathrm{NMR}(400 \mathrm{MHz}, \mathrm{DMSO}): 7.44(\mathrm{~m}, 1 \mathrm{H}), 7.08$ (s, 2H), $6.31(\mathrm{t}, 1 \mathrm{H}), 6.07(\mathrm{dd}, 1 \mathrm{H}), 4.87(\mathrm{~s}, 1 \mathrm{H}), 4.15-$ $4.11(\mathrm{~m}, 2 \mathrm{H}), 1.13(\mathrm{t}, 3 \mathrm{H}) .{ }^{13} \mathrm{C}-\mathrm{NMR}(100 \mathrm{MHz}, \mathrm{DMSO})$ : $161.3\left(\mathrm{CNH}_{2}\right), 158.7(\mathrm{C}=\mathrm{O}), 156.0(\mathrm{CNH}), 155.9$ (quat. Ar C), 142.4 (Ar C), 129.7 (Ar C), $120.6(\mathrm{CN}), 110.8$ (Ar C), 105.9 (quat. C), $61.4\left(\mathrm{CH}_{2}\right), 55.2$ (quat. C), $31.2(\mathrm{CH})$, $14.3\left(\mathrm{CH}_{3}\right)$.Anal.calc. (\%) for $\mathrm{C}_{14} \mathrm{H}_{12} \mathrm{~N}_{4} \mathrm{O}_{4}, \mathrm{C} 56.00, \mathrm{H}$ 4.03 Found: C 56.29, H 4.04.

(5m) Ethyl 6-amino-5-cyano-4-(thiophen-2-yl)-1,4-di hydropyrano[2,3-c]pyrazole-3-carboxylate. Following the above mentioned procedure, $5 \mathrm{~m}$ was isolated as a yellowish solid $65 \%$, m.p $205-207^{\circ} \mathrm{C}$. IR spectrum, $v$, $\mathrm{cm}^{-1}: 3402\left(\mathrm{NH}_{2}\right), 3256(\mathrm{NH}), 2203(\mathrm{CN}), 1729$ (COOEt), 1627 (C=C). ${ }^{1} \mathrm{H}-\mathrm{NMR}(400 \mathrm{MHz}, \mathrm{DMSO})$ : $7.28(\mathrm{~m}, 1 \mathrm{H}), 7.10(\mathrm{~s}, 2 \mathrm{H}), 6.88-6.86(\mathrm{~m}, 2 \mathrm{H}), 5.08(\mathrm{~s}$, $1 \mathrm{H}), 4.16-4.11(\mathrm{~m}, 2 \mathrm{H}), 1.13(\mathrm{t}, 3 \mathrm{H}) .{ }^{13} \mathrm{C}-\mathrm{NMR}(100$ $\mathrm{MHz}, \mathrm{DMSO}): 160.7\left(\mathrm{CNH}_{2}\right), 158.6(\mathrm{C}=\mathrm{O}), 155.4$ (CNH), 141.0 (quat. Ar C), 129.7 (Ar C), 127.1 (Ar C), 124.7 ( $\mathrm{Ar} \mathrm{C}), 120.7(\mathrm{CN}), 104.2$ (quat. C), $61.5\left(\mathrm{CH}_{2}\right)$, 58.2 (quat. C), $32.6(\mathrm{CH}), 14.3\left(\mathrm{CH}_{3}\right)$.Anal.calc. (\%) for $\mathrm{C}_{14} \mathrm{H}_{12} \mathrm{~N}_{4} \mathrm{O}_{3} \mathrm{~S}, \mathrm{C} 53.16, \mathrm{H} 3.82$ Found: C 54.35, H 3.61.

(5n) Ethyl 6-amino-5-cyano-4-ethyl-1,4-dihydropyrano [2,3-c]pyrazole-3-carboxylate. Following the above mentioned procedure, $5 \mathrm{n}$ was isolated as a yellowish solid $90 \%$, m.p $180-182{ }^{\circ} \mathrm{C}$. IR spectrum, $v, \mathrm{~cm}^{-1}: 3421$ $\left(\mathrm{NH}_{2}\right), 3178(\mathrm{NH}), 2192(\mathrm{CN}), 1712$ (COOEt), 1633 (C=C). ${ }^{1} \mathrm{H}-\mathrm{NMR}$ (400 MHz, DMSO): 6.91 (s, 1H), 4.29$4.22(\mathrm{~m}, 2 \mathrm{H}), 3.72(\mathrm{t}, 1 \mathrm{H}), 1.86-1.79(\mathrm{~s}, 1 \mathrm{H}), 1.69-1.59$ 
(m, 1H), 1.28-1.26 (t, 3H), 0.61-0.58 (t, 3H). ${ }^{13} \mathrm{C}-\mathrm{NMR}$ (100 MHz, DMSO): $162.2\left(\mathrm{CNH}_{2}\right), 159.0(\mathrm{C}=\mathrm{O}), 156.6$ (CNH), $121.2(\mathrm{CN}), 103.8$ (quat. C), 96.9 (quat. C), 61.6 $\left(\mathrm{CH}_{2}\right), 54.7$ (quat. C), $31.9(\mathrm{CH}), 28.0\left(\mathrm{CH}_{2}\right), 14.5\left(\mathrm{CH}_{3}\right)$, $8.78\left(\mathrm{CH}_{3}\right)$.Anal.calc. (\%) for $\mathrm{C}_{12} \mathrm{H}_{14} \mathrm{~N}_{4} \mathrm{O}_{3}, \mathrm{C} 54.96$, H 5.38 Found: C 53.85, H 5.57.

(50) Ethyl 6-amino-5-cyano-4-isopropyl-1,4-dihydro pyrano[2,3-c]pyrazole-3-carboxylate. Following the above mentioned procedure, 50 was isolated as a yellowish solid 91\%, m.p 200-201 ${ }^{\circ} \mathrm{C}$. IR spectrum, $v, \mathrm{~cm}^{-1}: 3427$ $\left(\mathrm{NH}_{2}\right), 3178(\mathrm{NH}), 2192(\mathrm{CN}), 1712$ (COOEt), 1633 $(\mathrm{C}=\mathrm{C}) .{ }^{1} \mathrm{H}-\mathrm{NMR}$ (400 MHz, DMSO): 6.98 (s, 2H), 4.30$4.21(\mathrm{~m}, 2 \mathrm{H}), 3.57(\mathrm{~d}, 1 \mathrm{H}), 2.00-1.97(\mathrm{~m}, 1 \mathrm{H}), 1.25(\mathrm{t}, 3 \mathrm{H})$, $0.93(\mathrm{t}, 3 \mathrm{H}), 0.57(\mathrm{t}, 3 \mathrm{H}) .{ }^{13} \mathrm{C}-\mathrm{NMR}(100 \mathrm{MHz}, \mathrm{DMSO})$ : $163.5\left(\mathrm{CNH}_{2}\right), 159.1(\mathrm{C}=\mathrm{O}), 156.9(\mathrm{CNH}), 128.7$ (quat. C), $122.3(\mathrm{CN}), 105.2$ (quat. C), $61.5\left(\mathrm{CH}_{2}\right), 51.6$ (quat. C), $37.6(\mathrm{CH}), 35.4(\mathrm{CH}), 20.7,17.1\left(\mathrm{CH}_{3}\right)$.Anal.calc. (\%) for $\mathrm{C}_{13} \mathrm{H}_{16} \mathrm{~N}_{4} \mathrm{O}_{3}, \mathrm{C} 56.51, \mathrm{H}$ 5.84 Found: C 56.18, H 5.85.

(5p) Ethyl 6-amino-5-cyano-4-heptyl-1,4-dihydropyrano [2,3-c]pyrazole-3-carboxylate. Following the above mentioned procedure, $5 \mathrm{p}$ was isolated as a yellowish solid $11 \%$, m.p 204-206 ${ }^{\circ} \mathrm{C}$. IR spectrum, $v, \mathrm{~cm}^{-1}: 3427\left(\mathrm{NH}_{2}\right)$, $3178(\mathrm{NH}), 2192(\mathrm{CN}), 1712$ (COOEt), $1633(\mathrm{C}=\mathrm{C}) .{ }^{1} \mathrm{H}-$ NMR (400 MHz, DMSO): 6.88 (s, 2H), 4.32-4.26 (m, 2H), $3.70(\mathrm{t}, 1 \mathrm{H}), 1.75(\mathrm{~m}, 1 \mathrm{H}), 1.56(\mathrm{~m}, 1 \mathrm{H}), 1.27(\mathrm{t}, 3 \mathrm{H}), 1.16$ (m, 10H), 0.83 (t, 3H). ${ }^{13} \mathrm{C}-\mathrm{NMR}$ (100 MHz, DMSO): 162.0 $\left(\mathrm{CNH}_{2}\right), 159.0(\mathrm{C}=\mathrm{O}), 156.4(\mathrm{CNH}), 129.0$ (quat. C), 121.2 (CN), 104.5 (quat. C), $61.5\left(\mathrm{CH}_{2}\right), 55.2$ (quat. C), 35.8 $(\mathrm{CH}), 31.6(\mathrm{CH}), 31.1\left(\mathrm{CH}_{2}\right), 29.3\left(\mathrm{CH}_{2}\right), 29.0\left(\mathrm{CH}_{2}\right), 24.0$ $\left(\mathrm{CH}_{2}\right), 22.5\left(\mathrm{CH}_{2}\right), 14.5\left(\mathrm{CH}_{3}\right), 14.4\left(\mathrm{CH}_{3}\right)$.Anal.calc. (\%) for $\mathrm{C}_{17} \mathrm{H}_{24} \mathrm{~N}_{4} \mathrm{O}_{3}, \mathrm{C} 61.43, \mathrm{H}$ 7.28 Found: C 61.44, H 7.22.

(5q) Ethyl 6'-amino-5'-cyano-2-oxo-1'H-spiro[indoline3,4'-pyrano[2,3-c]pyrazole]-3'-carboxylate. Following the above mentioned procedure, $5 \mathrm{q}$ was isolated as a yellowish solid 69\%, m.p $270-271^{\circ} \mathrm{C}$. IR spectrum, $v, \mathrm{~cm}^{-1}: 3371$ $\left(\mathrm{NH}_{2}\right), 3165(\mathrm{NH}), 2187(\mathrm{CN}), 1713$ (COOEt), 1648 $(\mathrm{C}=\mathrm{C}) .{ }^{1} \mathrm{H}-\mathrm{NMR}(400 \mathrm{MHz}, \mathrm{DMSO}): 0.86-0.89(\mathrm{t}, 3 \mathrm{H})$, 3.85-3.88 (q, 2H), 6.81-6.92 (m, 3H), 7.13-7.15 (m, 1H), 7.25 (s, 2H), 10.55 (s, 1H). ${ }^{13} \mathrm{C}-\mathrm{NMR}(100 \mathrm{MHz}, \mathrm{DMSO})$ : $178.0(\mathrm{C}=\mathrm{O}), 161.5\left(\mathrm{CNH}_{2}\right), 158.1(\mathrm{C}=\mathrm{O}), 142.6(\mathrm{CNH})$, 134.6 ( $\mathrm{ArC}$ ), 129.0 ( $\mathrm{ArC}$ ), 124.2 ( $\mathrm{ArC}$ ), $122.6(\mathrm{CN}), 118.5$ (Ar C), 109.8 (quat. C), 100.6 (quat. C), $61.3(\mathrm{CH} 2), 57.3$ (quat. C), 48.0 (quat. C), $14.0\left(\mathrm{CH}_{3}\right)$.Anal.calc. (\%) for $\mathrm{C}_{17} \mathrm{H}_{13} \mathrm{~N}_{5} \mathrm{O}_{4}, \mathrm{C} 58.12$, H 3.73 Found: C 57.89, H 3.68.

(5r) Ethyl 6-amino-5-cyano-5'-(4-methoxyphenyl)-1'methyl-2'-oxo-1H-spiro[pyrano[2,3-c]pyrazole-4,3'pyrrolidine]-3-carboxylate. Following the above mentioned procedure, $5 \mathrm{r}$ was isolated as a yellowish solid 26\%, m.p 212-214 ${ }^{\circ} \mathrm{C}$. IR spectrum, $v, \mathrm{~cm}^{-1}: 3366$ $\left(\mathrm{NH}_{2}\right), 3193(\mathrm{NH}), 2193(\mathrm{CN}), 1733$ (COOEt), 1672 $(\mathrm{C}=\mathrm{C}) .{ }^{1} \mathrm{H}-\mathrm{NMR}$ (400 MHz, DMSO): $1.26-1.30(\mathrm{t}, 3 \mathrm{H})$, 1.96-2.01 (dd, $1 \mathrm{H}), 2.51$ (s, 3H), 2.83-2.86 (dd, $1 \mathrm{H}), 3.72$ (s, 3H), 4.31-4.33 (q, 2H), 4.63-4.67 (t, 1H), 6.89-6.92 (m, 2H), 7.09 (s, 2H), 7.33-7.35 (d, 2H). ${ }^{13} \mathrm{C}-\mathrm{NMR}(100$ MHz, DMSO): $173.8(\mathrm{C}=\mathrm{O}), 161.0\left(\mathrm{CNH}_{2}\right), 159.4$ (quat. Ar C), 158.5 (C=O), 155.6 (quat. C), 133.8 (quat. Ar C), 128.9 (quat. C), 128.0 ( $\mathrm{Ar} \mathrm{C}), 120.5(\mathrm{CN}), 114.6(\mathrm{Ar} \mathrm{C})$, 105.2 (quat. $\mathrm{C}$ ), $62.0\left(\mathrm{CH}_{2}\right), 61.3(\mathrm{CH}), 60.9$ (quat. $\mathrm{C}$ ), $55.6\left(\mathrm{NCH}_{3}\right), 40.4\left(\mathrm{CH}_{2}\right), 40.2$ (quat. C), $29.3\left(\mathrm{OCH}_{3}\right)$, $14.6\left(\mathrm{CH}_{3}\right)$.Anal.calc. (\%) for $\mathrm{C}_{21} \mathrm{H}_{21} \mathrm{~N}_{5} \mathrm{O}_{5}, \mathrm{C} 59.57, \mathrm{H}$ 5.00 Found: C 59.72, H 5.20.

(5s) Ethyl 6-amino-5-cyano-2', 3', 5',6'-tetrahydro-1Hspiro[pyrano[2,3-c] pyrazole-4,4'-thiopyran]-3-carboxy late. Following the above mentioned procedure, $5 \mathrm{~s}$ was isolated as a yellowish solid 19\%, m.p $190-191{ }^{\circ} \mathrm{C}$. ${ }^{1} \mathrm{H}$ NMR (400 MHz, DMSO-D6): $\delta 6.85\left(\mathrm{~s}, \mathrm{NH}_{2}\right), 4.31$ (q, $2 \mathrm{H}), 3.48(\mathrm{td}, 2 \mathrm{H}), 2.69-2.60(\mathrm{td}, 2 \mathrm{H}), 2.40(\mathrm{~d}, 2 \mathrm{H}), 1.92$ (d, 2H), $1.31(\mathrm{t}, 3 \mathrm{H}) ;{ }^{13} \mathrm{C}-\mathrm{NMR}$ (100 MHz DMSO): 161.5 $\left(\mathrm{CNH}_{2}\right), 158.7(\mathrm{C}=\mathrm{O}), 154.6(\mathrm{CNH}), 129.1$ (quat. C), $124.5(\mathrm{CN}), 110.3$ (quat. C), $61.9\left(\mathrm{CH}_{2}\right), 59.0$ (quat. C), 36.8 (quat $\mathrm{C}$ ), $33.2\left(2 \mathrm{XCH}_{2}\right), 23.4\left(2 \mathrm{XCH}_{2}\right), 14.63$ $\left(\mathrm{CH}_{3}\right)$.Anal.calc. (\%) for $\mathrm{C}_{14} \mathrm{H}_{16} \mathrm{~N}_{4} \mathrm{O}_{3} \mathrm{~S}, \mathrm{C} 52.49$, H 5.03 Found: C 52.49, H 5.07.

(5t) Ethyl 6'-amino-5'-cyano-1-methyl-1'H-spiro[pipe ridine-4,4'-pyrano[2,3-c]pyrazole]-3'-carboxylate [5t]. Following the above mentioned procedure, 5t was isolated as a yellowish solid 23\%, m.p $200-202{ }^{\circ} \mathrm{C}$. ${ }^{1} \mathrm{H}$ NMR (400 MHz, DMSO-D6): $\delta 6.80\left(\mathrm{~s}, \mathrm{NH}_{2}\right), 4.30$ (q, $2 \mathrm{H}), 3.45(\mathrm{td}, 2 \mathrm{H}), 3.01\left(\mathrm{~s}, \mathrm{CH}_{3}\right), 2.69-2.60(\mathrm{td}, 2 \mathrm{H}), 2.40$ (d, 2H), $1.92(\mathrm{~d}, 2 \mathrm{H}), 1.31(\mathrm{t}, 3 \mathrm{H}) ;{ }^{13} \mathrm{C}-\mathrm{NMR}(100 \mathrm{MHz}$ DMSO): $161.5\left(\mathrm{CNH}_{2}\right), 158.7(\mathrm{C}=\mathrm{O}), 154.6(\mathrm{CNH})$, 129.1 (quat. C), $124.5(\mathrm{CN}), 110.3$ (quat. C), $61.9\left(\mathrm{CH}_{2}\right)$, 59.0 (quat. C), 36.8 (quat. C), $35.8\left(\mathrm{CH}_{3}\right), 33.2\left(2 \mathrm{XCH}_{2}\right)$, $23.4\left(2 \mathrm{XCH}_{2}\right), 14.63\left(\mathrm{CH}_{3}\right)$. 


\section{- RESULTS AND DISCUSSION}

First dihydropyrano[2,3-c]pyrazole-3-carboxylate type compounds were successfully reported by Gein et al. via one-pot two-parallel reaction manners [13]. These reactions proceeded by short boiling of aromatic aldehydes and malononitrile to give intermediates of arylidenemalonodinitrile. Subsequent addition of the preformed pyrazolone upon reacting hydrazine hydrate, diethyl oxaloacetate, and acetic acid onto the prepared reaction of aromatic aldehydes and malononitrile led to the cyclized products from moderate to excellent yield. Replicating the exact Gein's reaction protocol and using similar of hydrazine hydrate, diethyl oxalacetate, benzaldehyde and malononitrile as our reaction model indeed furnished us the cyclized product $\mathbf{5 a}$ but in a reasonable yield of $35 \%$. Attempts on optimization by changing the reaction solvent from mono- to biphasic solvent systems also led to similar reasonable yields (Table 1, Entries 1-6) [11]. Realizing the complexity of the previous reaction protocol we then subjected our reaction model employing domino fashion type of reaction in acidic ethanolic solution which surprisingly furnished us with the cyclized product $\mathbf{5 a}$ in $82 \%$ yield (Table 1, Entry 7) (Scheme 1, Method A). The fact that addition of selected known MCRs catalysts as towards optimization steps do not contribute to much higher yield indicates that this catalyst-free domino type MCRs was already performed optimally (Table 1, Entries 8-11).
To probe the generality of this methodology, this method was then extended using a variety of carbonyl functionalities (aromatic, aliphatic, heteroaromatic and diketo compounds). The details of the reaction protocol and the product structure of dihydropyrano[2,3c]pyrazole-3-carboxylates (5a-5t) are depicted in Table 2 (Scheme 1, method A). The nature of the substituents on the aromatic ring of the aldehydes displayed a significant effect on the product yields. Aromatic aldehydes bearing electron-withdrawing groups such as nitro, cyano, and halogens (Table 2, Entries 5-11) undoubtedly contributed to much higher yields (74$83 \%)$ of the cyclized products as compared to those with electron-donating groups (60-69\%) (Table 2, Entries 24). This was due to the induced electrophilicity of the aromatic aldehydes from the electron withdrawing group of compounds $\mathbf{5 e - 5 h}$. Likewise, heteroaromatic aldehydes of furan-2-carbaldehyde and thiophene-2carbaldehyde also readily underwent the same sequence of reactions yielding their respected cyclized products in reasonable of 63 and 65\% yields (Table 2, Entries 12-13).

Previously, many aliphatic aldehydes were reported not ready to be used as electrophiles in one-pot reaction procedures due to their tendency to undergo selfcondensation or Cannizzaro-type reactions [19]. Nevertheless, via our domino-type reaction method, alkylated-pyranopyrazole-type compounds were successfully synthesized by using propanal and 2-methyl propanol in excellent yields (Table 2, Entries 14-15).

Table 1. Synthesis of $\mathbf{5 a}$ in different solvents and reaction conditions

\begin{tabular}{ccccc}
\hline Entry & Solvent & Time (min) & Catalyst & Yield (\%) \\
\hline 1 & methanol & 90 & - & $18^{\mathrm{a}}$ \\
2 & water & 90 & - & - \\
3 & ethanol & 90 & - & $29^{\mathrm{a}}$ \\
4 & ethanol:water (1:1) & 90 & - & - \\
5 & ethanol:water (9:1) & 90 & - & $18^{\mathrm{a}}$ \\
6 & ethanol:acetic acid & 90 & - & $35^{\mathrm{a}}$ \\
7 & ethanol:acetic acid & 30 & - & $82^{\mathrm{b}}$ \\
8 & ethanol:acetic acid & 30 & Triethylamine & $82^{\mathrm{b}}$ \\
9 & ethanol:acetic acid & 30 & Potassium carbonate & $84^{\mathrm{b}}$ \\
10 & ethanol:acetic acid & 30 & Ammonium acetate & $86^{\mathrm{b}}$ \\
11 & ethanol:acetic acid & 30 & Piperidine & $87^{\mathrm{b}}$ \\
\hline
\end{tabular}

${ }^{a}$ Gein's four-component two-parallel reaction manner, ${ }^{b}$ Domino one-pot four-component reaction manners 
Table 2. Synthesis of dihydropyrano[2,3-c]pyrazole-3-carboxylates (5a-t)

Yield (\%)
Method A/B


Table 2. Synthesis of dihydropyrano[2,3-c]pyrazole-3-carboxylates (5a-t) (Continued)

\begin{tabular}{|c|c|c|c|c|}
\hline Entry & Product & $\begin{array}{l}\text { Time } \\
(\min )\end{array}$ & $\begin{array}{c}\text { Yield (\%) } \\
\text { Method A/B }\end{array}$ & $\begin{array}{l}\text { m.p } \\
\left({ }^{\circ} \mathrm{C}\right)\end{array}$ \\
\hline 9 & & 30 & $73^{b}$ & $224-225$ \\
\hline 10 & & 30 & $50^{\mathrm{b}}$ & $228-229$ \\
\hline 11 & & 30 & $10^{\mathrm{a}}, 74^{\mathrm{b}}$ & $218-219$ \\
\hline 12 & & 30 & $22^{\mathrm{a}}, 63^{\mathrm{b}}$ & $216-218$ \\
\hline 13 & & 30 & $18^{\mathrm{a}}, 65^{\mathrm{b}}$ & 205-207 \\
\hline 14 & & 30 & $90^{\mathrm{b}}$ & $180-182$ \\
\hline 15 & & 30 & $5^{\mathrm{a}}, 91^{\mathrm{b}}$ & $190-192$ \\
\hline 16 & & 30 & $5^{\mathrm{a}}, 11^{\mathrm{b}}$ & $200-203$ \\
\hline
\end{tabular}


Table 2. Synthesis of dihydropyrano[2,3-c]pyrazole-3-carboxylates (5a-t) (Continued)

Entry $\begin{gathered}\text { Time } \\ (\mathrm{min})\end{gathered} \begin{gathered}\begin{array}{c}\text { Yield }(\%) \\ \text { Method A/B }\end{array} \\ \left({ }^{\circ} \mathrm{C}\right)\end{gathered}$

In spite of that, the product yield of an alkylatedpyranopyrazole was observed to decrease significantly upon increasing the aliphatic chain length (Table 2, Entry 16). Further advance of non-optimized MCRs reaction by employing diketo compounds had also successfully furnished us the interesting novel spiropyrano-pyrazole carboxylate type of compounds in reasonable yields (Table 2, Entries 17-20).

In a different mechanistic study, a reverse domino one-pot reaction manner was also performed in which arylidenemalonodinitrile intermediates, 7 were synthesized prior to reacting malononitrile and an aromatic aldehyde. Later hydrazine and diethyl oxaloacetate were added onto the reactions. Interestingly, such reverse domino one-pot reaction manner failed to furnish high yields of the cyclized products $(5-37 \%)$ as many unwanted side products were observed from the T.L.C (Scheme 1, Method B) (Table 2, Entries 1-3, 5, 7-8, 11-13, 15-16).

Based on the above findings, the best plausible mechanism for the synthesis of (5) is proposed (Scheme 2).
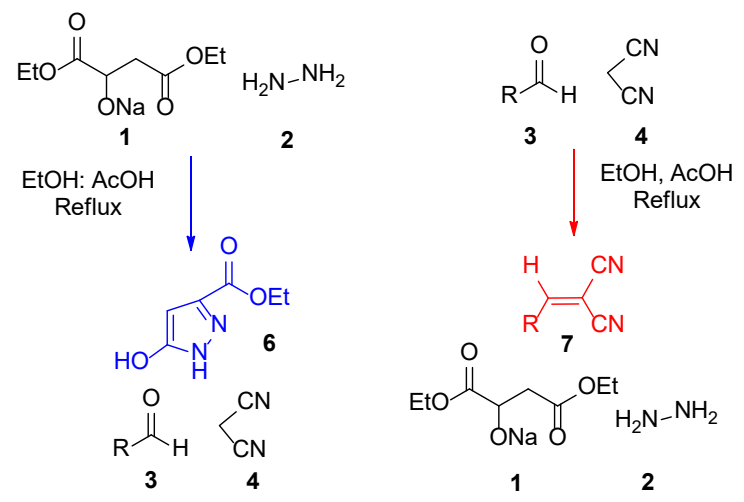

${ }_{\mathrm{R}}^{\mathrm{H}}={ }_{\mathrm{CN}}^{\mathrm{CN}}$<smiles>CCOC(=O)CC(O[N+](=O)[O-])C(=O)OCC</smiles>
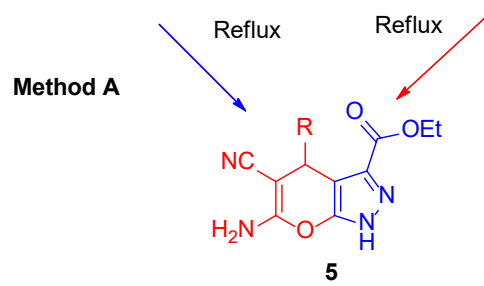

Method B

Scheme 1. Different approaches in synthesizing dihydropyrano[2,3-c]pyrazoles (5)

Pyrazolone (A) was initially formed upon condensation of the diethyl oxaloacetate salt with hydrazine, followed by intramolecular nucleophilic cyclization and elimination 

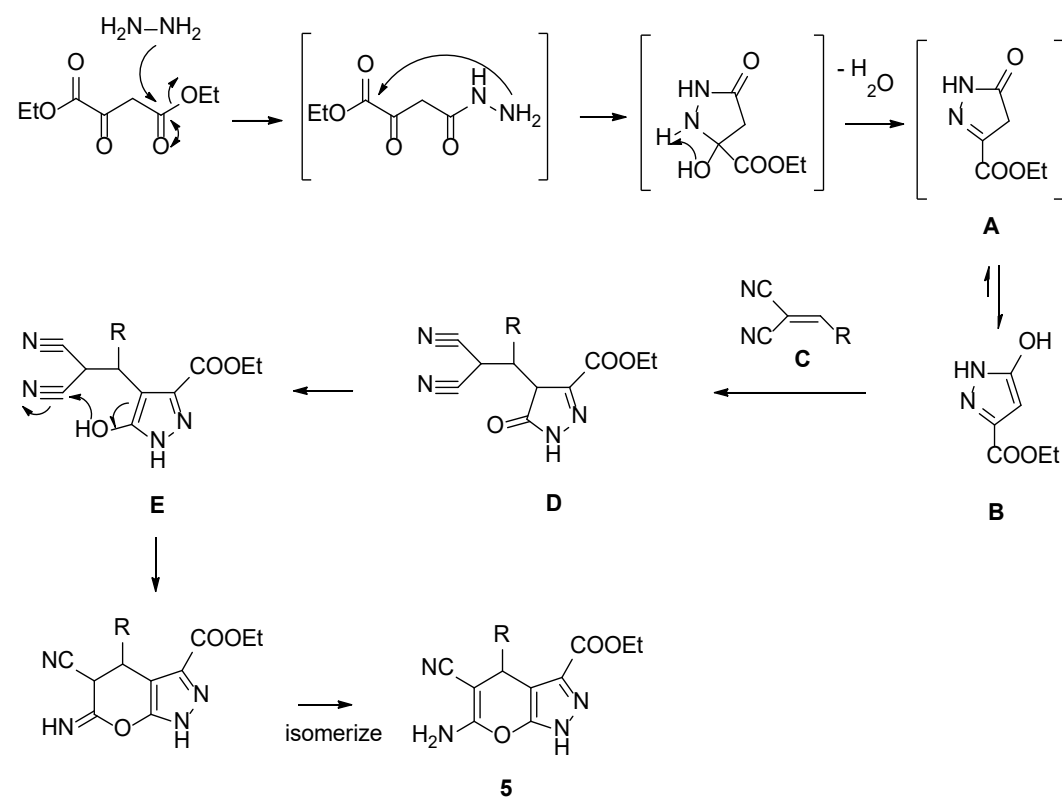

Scheme 2. Plausible mechanism for the synthesis of 5(a-t)

Table 3. Antimicrobial screening results

\begin{tabular}{ccccccc}
\hline \multirow{2}{*}{ Compound } & \multicolumn{5}{c}{ Gram -ve } & \multicolumn{5}{c}{ Gram +ve } \\
\cline { 2 - 7 } $5 \mathrm{a}$ & E.C & S.T & P.V & S.A & S.C & S.H \\
\hline $5 \mathrm{~b}$ & - & - & - & - & - & - \\
$5 \mathrm{c}$ & - & - & - & - & - & - \\
$5 \mathrm{~d}$ & - & - & - & - & - & - \\
$5 \mathrm{e}$ & - & - & - & - & - & - \\
$5 \mathrm{f}$ & - & - & - & - & - & - \\
$5 \mathrm{~g}$ & - & - & - & - & - & - \\
$5 \mathrm{~h}$ & - & - & - & - & - & - \\
$5 \mathrm{i}$ & - & - & - & - & - & - \\
$5 \mathrm{j}$ & - & - & - & - & - & - \\
$5 \mathrm{k}$ & - & - & - & - & - & - \\
$5 \mathrm{l}$ & - & - & - & - & - & - \\
$5 \mathrm{~m}$ & - & - & - & - & - & - \\
$5 \mathrm{n}$ & - & - & 14.0 & - & 10.5 & 17.0 \\
$5 \mathrm{c}$ & - & - & - & - & - & - \\
$5 \mathrm{p}$ & - & - & - & - & - & - \\
\hline +ve Control & 17.0 & 19.0 & 16.5 & 18.0 & 22.0 & 19.0 \\
-ve Control & - & - & - & - & - & - \\
\hline Values are mean inhibition zone (mm); No inhibition (-) & & & \\
E.C $=$ Escherichia coli, S.T = Salmonella typhimurium, P.V = Proteus vulgaris, S.A = Staphylococcus \\
aureus, S.C $=$ Staphylococcus cohnii (clinical strain), S.H $=$ Staphyloccoccus haemolyticus.
\end{tabular}

of water. Pyrazolone (A) then underwent tautomerization to a more active enolicpyrazolone (B) in which subsequent reaction with pre-synthesized aryl/alkylidene malonodinitriles (C) furnished intermediates (D) via Michael-type reaction. Finally, intermediates (D) isomerized to compounds (E), which then underwent 
Thorpe-Ziegler intramolecular cyclization to yield the final products of dihydropyrano[2,3-c]pyrazol-3-carboxylates (5). In every step of the synthetic transformation, acetic acid was anticipated to play a significant role in increasing the solubility of the diethyl oxaloacetate sodium salt in ethanol and also as a proton donor.

As for antimicrobial screening, study shows that the presence of thiophene ring in the structure of $5 \mathrm{~m}$ enhances its activity against Proteus vulgaris (P.V), Staphylococcus cohnii (S.C), and Staphylococcus haemolyticus (S.H). It was also reasoned that limited solubility for most of the compound also contributed to this low antimicrobial activity properties for other pyrano-pyrazole analogs (Table 3 ).

\section{- CONCLUSION}

In summary, a salient reaction protocol using domino one-pot, four-component approach towards generating pyranopyrazole-carboxylate type compounds has been developed. This protocol was found applicable for both aromatic and aliphatic aldehydes which make it a useful for the synthesis of a different class of pyranopyrazoles under green catalyst-free MCRs reaction. Nevertheless, biological screening of this spiropyranopyrazole type compounds revealed limited potential of these compounds for antimicrobial agents.

\section{- ACKNOWLEDGMENTS}

The authors would like to acknowledge Universiti Teknologi MARA and Malaysian Government (MOHE) for the financial support (600-IRMI/FRGS 5/3 (0071/2016).

\section{- REFERENCES}

[1] Mistry, P.T., Kamdar, N.R., Haveliwala, D.D., and Patel, S.K., 2012, Synthesis, characterization, and invitro biological studies of some novel pyran fused pyrimidone derivatives, J. Heterocycl. Chem., 49 (2), 349-357.

[2] Kuo, S.C., Huang, L.J., and Nakamura, H., 1984, Studies on heterocyclic compounds. 6. Synthesis and analgesic and antiinflammatory activities of 3,4dimethylpyrano[2,3-c]pyrazol-6-one derivatives, $J$. Med. Chem., 27 (4), 539-544.
[3] Ahluwalia, V.K., Dahiya, A., and Garg, V., 1997, Reaction of 5-amino-4-formyl-3-methyl(or phenyl)1-phenyl-1H-pyrazoles with active methylene compounds: Synthesis of fused heterocyclic rings, Indian J. Chem., Sect B, 36 (1), 88-90.

[4] Wang, J.L., Liu, D., Zheng, Z.J., Shan, S., Han, X., Srinivasula, S.M., Croce, C.M., Alnemri, E.S., and Huang, Z., 2009, Structure-based discovery of an organic compound that binds Bcl-2-protien and induces apoptosis of tumor cell, Proc. Natl. Acad. Sci. U.S.A., 97 (13), 7124-7129.

[5] Mandha, S.R., Siliveri, S., Alla, M., Bommena, V.R., Bommineni, M.R., and Balasubramanian, S., 2012, Eco-friendly synthesis and biological evaluation of substituted pyrano[2,3-c]pyrazoles, Bioorg. Med. Chem. Lett., 22 (16), 5272-5278.

[6] Foloppe, N., Fisher, L.M., Howes, R., Potter, A., Robertson, A.G.S., and Surgenor, A.E., 2006, Identification of chemically diverse Chk1 inhibitors by receptor-based virtual screening, Bioorg. Med. Chem., 14 (14), 4792-4802.

[7] Ramiz, M.M.M., Hafiz, I.S.A., Rahim, M.A.M.A., and Gaber, H.M., 2012, Pyrazolones as building blocks in heterocyclic synthesis: Synthesis of new pyrazolopyran, pyrazolopyridazine and pyrazole derivatives of expected antifungicidal activity, $J$. Chin. Chem. Soc., 59 (1), 72-80.

[8] Kiyani, H., Samimi, H.A., Ghorbani, F., and Esmaieli, S., 2013, One-pot, four-component synthesis of pyrano[2,3-c]pyrazolescatalyzed by sodium benzoate in aqueous medium, Curr. Chem. Lett., 2 (4), 197-206.

[9] Otto, S., and Engberts, J.B.F.N., 2000, Diels Alder reactions in water, Pure Appl. Chem., 72 (7), 13651372.

[10] Shestopalov, A.M., Emeliyanova, Y.M., Shestopalov, A.A., Rodinovskaya, L.A., Niazimbetova, Z.I., and Evans, D.H., 2003, Cross-condensation of derivatives of cyanoacetic acid and carbonyl compounds. Part 1: Single-stage synthesis of $1^{\prime}$-substituted 6-aminospiro-4-(piperidine-4')-2H,4H-pyrano[2,3-c]pyra zole-5-carbonitriles, Tetrahedron, 59 (38), 7491-7496. 
[11] Zonouz, A.M., Eskandari, I., and Khavasi, H.R., 2012, A green and convenient approach for the synthesis of methyl 6-amino-5-cyano-4-aryl-2,4-dihydropyrano [2,3-c]pyrazole-3-carboxylates via a one-pot, multicomponent reaction in water, Tetrahedron Lett., 53 (41), 5519-5522.

[12] Weber, L., 2002, Multi-component reactions and evolutionary chemistry, Drug Discovery Today, 7 (2), 143-147.

[13] Gein, V.L., Zamaraeva, T.M., and Kozulina, I.V., 2014, New synthesis of ethyl 6-amino-4-aryl-5cyano-1,4-dihydropyrano[2,3-c]pyrazole-3-carboxy lates, Russ. J. Org. Chem., 50 (5), 691-693.

[14] Kumler, W.D., Kun, E., and Shoolery, J.N., 1962, The enolization of oxaloacetic acid, diethyl oxaloacetate, and diethyl fluorooxaloacetate as determined by NMR analyses, J. Org. Chem., 27 (4), 1165-1167.

[15] Mohammat, M.F., Shaameri, Z., and Hamzah, A.S., 2009, Synthesis of 2,3-dioxo-5-(substituted) arylpyrroles and their 2-oxo-5-aryl-3-hydrazone pyrrolidine derivatives, Molecules, 14 (1), 250-256.
[16] Mohammat, M.F., Najim, N., Mansor, N.S., Sarman, S., Shaameri, Z., Zain, M.M., and Hamzah, A.S., 2011, Synthesis and bioactivity of some 2-oxo5-aryl-3-hydrazone and 2-oxo-5-aryl-4-hydrazone pyrrolidine derivatives, Arkivoc, 2011 (9), 429-438.

[17] Johari, S.A., Mohtar, M., Syed Mohammad, S.A., Mohammat, M.F., Sahdan, R., Mohamed, A., and Mohamad Ridhwan, M.J., 2017, In vitro evaluations and in vivo toxicity and efficacy studies of MFM501 against MRSA, Biomed Res. Int., 2017, 8032865.

[18] Johari, S.A., Mohtar. M., Syed Mohammad. S.A., Sahdan. R., Shaameri. Z., Hamzah, A.S., and Mohammat, M.F., 2016, In vitro inhibitory and cytotoxic activity of MFM 501, a novel codonopsinine derivative, against methicillinresistant Staphylococcus aureus clinical isolates, Biomed Res. Int., 2015, 823829.

[19] Khalil, T., Manouchehr, M., Nosrat, O.M., and Elahe, K., 2009, Ruthenium-catalyzed cross aldol reaction with aldehydes and ketones, Arkivoc, 2009 (2) 68-75. 